\title{
Changes in Speaking Fundamental Frequency Characteristics with Aging
}

\author{
Masaki Nishio $^{a}$ Seiji Niimi ${ }^{b}$ \\ a Department of Speech Therapy, Language and Hearing Sciences, School of Health Sciences, Niigata University of \\ Health and Welfare, Niigata, and ${ }^{b}$ Department of Speech and Hearing Science, International University of Health \\ and Welfare, Ohtawara, Japan
}

\section{Key Words}

Speaking fundamental frequency, reference intervals •

Fundamental frequency · Aging · Acoustic analysis

\begin{abstract}
Changes in speaking fundamental frequency (SFF) associated with aging were studied in a total of 374 healthy normal speakers (187 males and 187 females) from adolescent to older age groups. Participants were asked to read a sample passage aloud, and acoustic analysis was performed. The main results were as follows: (1) Males exhibited no significant trend for SFF changes in aging. However, a slight increase was observed in participants aged 70 years or older. (2) Females in their 30s and 40s showed obviously lower frequencies than those in their 20s. Across all age groups, including the 80s, SFF tended to decrease markedly in association with aging. (3) The degree of SFF change in association with aging was much larger in females than in males. In addition, reference intervals (mean $\pm 1.96 \mathrm{SD}$ ) obtained for males and females in each age group are considered useful for clinical detection of abnormalities of SFF, as well as for detection of laryngeal diseases causing SFF abnormality.
\end{abstract}

Copyright $\odot 2008$ S. Karger AG, Basel
(C) 2008 S. Karger AG, Base

$1021-7762 / 08 / 0603-0120 \$ 24.50 / 0$

Fax +41613061234

E-Mail karger@karger.ch

www.karger.com
Accessible online at:

www.karger.com/fpl

\section{Introduction}

Although numerous studies have been conducted on fundamental frequency of the voice, the results are diverse and inconsistent. One reason for this is differences in methodology. Another reason may involve differences in the precision of the experimental environment used for recording and analysis, including performance of the instruments used and skill of the experimenters. An inadequate experimental environment might occasionally lead to spurious high-frequency readings. In addition, to our knowledge, only five of the numerous acoustic studies of speaking fundamental frequency (SFF) have examined reliability [1-5].

Many of the previous reports on SFF measurement in adult males by an acoustic technique, using a passage or sentence, have found that SFF is higher at an advanced age than in youth [1,4-8]. However, Benjamin [9] and Guimarães and Abberton [10] reported that SFF was lower in their elderly sample population, while Ramig and Ringel [2] reported that SFF in their elderly group was slightly, but not significantly, higher than that in their younger group. Hollien and Shipp [6], Pegoraro Krook [4], and Brown et al. [8] reported that SFF decreases transiently in middle age and increases again at an advanced age.

Masaki Nishio, $\mathrm{PhD}$

Department of Speech Therapy, Language and Hearing Sciences

School of Health Sciences, Niigata University of Health and Welfare

Shimami-cho 1398, Niigata 950-3198 (Japan)

Tel./Fax +81 25257 4431, E-Mail nishio@nuhw.ac.jp 
Similar acoustic studies of SFF have been conducted in adult females, using a passage, sentence or picture description to obtain speech samples. The results of previous studies are highly consistent in that SFF decreased with advancing age [3, 4, 7-16]. However, considerable differences are seen in the degree of change in SFF associated with aging among investigators. Stoicheff [12], Morgan and Rastatter [3], Pegoraro Krook [4], and Awan [16] reported that SFF decreases in the 40s-60s, then slightly increases again above the age of 70 . Thus, consensus has not been achieved with respect to some aspects of SFF and its relationship to age in males and females, and further studies are still needed.

In the present study, we collected and analyzed information related to SFF for a relatively large population with the aim of clarifying the following issues: (1) changes in SFF characteristics with age in males throughout adulthood; (2) changes in SFF characteristics with age in females throughout adulthood, and (3) reference interval or normal range (mean \pm 1.96 standard deviation) of SFF in males and females during youth, middle age and advanced age.

It has long been known that the fundamental frequency of the human voice reflects the physiological functions of vocal folds [17-20], and its measurement is noninvasive and easy. Therefore, the findings of the present study are believed to be useful for clinically determining not only SFF abnormalities and the degree of abnormality but also laryngeal diseases that can cause SFF abnormalities.

\section{Methods}

\section{Participants}

The participants were 374 native speakers of Japanese (187 males, 187 females) who were indicated as healthy according to a questionnaire (Appendix), and had neither laryngeal pathology nor history of neurological disease. Additional criteria for selection were no history of professional voice training and passing both hearing and visual screening tests. When a pathological voice feature was perceived, despite the participants reporting no laryngeal dysfunction, a laryngoscopic examination was performed by an otorhinolaryngologist (one of the authors of the current study, S.N.). Individuals found to have laryngeal dysfunction, such as a vocal fold polyp, at laryngoscopy, were excluded from the study.

Smoking habits were not evaluated as inclusion criteria since one of the major objectives of this study was to determine the reference interval of SFF for typical speakers, and the total percentage of smokers among Japanese males 20 years or older averaged $82.3,70.2,58.8$ and $46.9 \%$ in $1965,1980,1995$ and 2004, respectively, according to the 'National Survey of the Percentage of Tobacco Smokers' conducted by Japan Tobacco Inc. [21]. Although
Table 1. Means and standard deviations of participants by age group

\begin{tabular}{llll}
\hline Groups & $\mathrm{n}$ & Mean & SD \\
\hline Young & & & \\
$\quad$ Males & 77 & 23.13 & 3.57 \\
$\quad$ Females & 77 & 22.88 & 3.61 \\
Middle-aged & & & \\
$\quad$ Males & 55 & 48.62 & 6.46 \\
$\quad$ Females & 55 & 48.45 & 5.27 \\
Elderly & & & \\
$\quad$ Males & 55 & 69.55 & 6.23 \\
$\quad$ Females & 55 & 70.75 & 6.61 \\
\hline
\end{tabular}

a decreasing tendency in smoking incidence can be observed over time, the percentage remains high. In particular, a very large percentage (about $80 \%$ ) of elderly people had smoked at some time in the past. Therefore, exclusion of smokers from the participants might have produced results that would fail to reflect the general traits of elderly males and possibly even bias the reference value considerably.

Participants were divided into three groups according to age (table 1): the young group consisted of 77 males and 77 females aged between 19 and 34 years; the middle-aged group consisted of 55 males and 55 females aged between 35 and 59 years, and the elderly group consisted of 55 males and 55 females aged 60 years and older. As no general consensus has been reached on age division in adulthood, the present study followed Santrock's [22] study.

\section{Recording Procedure}

The speech sample employed to measure SFF was a standard passage, 'The North Wind and the Sun', in Japanese which is 223 morae in length. Japanese is classified as a 'moric language' or a 'mora-counting language' and shows isochronism over mora units, whereas such languages as English, Russian, German, and Hungarian are 'syllabic languages' or 'syllable-counting languages' [23]. Thus, the mora was used as a measurement unit in the present study.

Participants were asked to read the passage once at a habitual and comfortable pitch, loudness, and speaking rate. All speech samples were recorded in a sound-treated studio on a digital audio-cassette recorder (Sony, PCMR500) with a sampling rate of $44.1 \mathrm{kHz}$. Before the recording, each participant was given an opportunity to practice reading the passage for familiarization with the speech protocol. If the recording was not successful, the participants were asked to participate in additional trials. A microphone (Shure, SM48) was positioned to maintain a constant mouth-to-microphone distance of $15 \mathrm{~cm}$.

\section{Data Analysis}

SFF measures were calculated for all sentences of the aforementioned passage using the Frequency Analysis Program of the Computerized Speech Lab (CSL) system (CSL, Kay Elemetrics, model 4400) operating on a desktop computer (Kay Elemetrics, Generic Windows PC, model 4307). CSL is a highly advanced 
Fig. 1. Comparison of mean SFFs among age groups (male speakers).

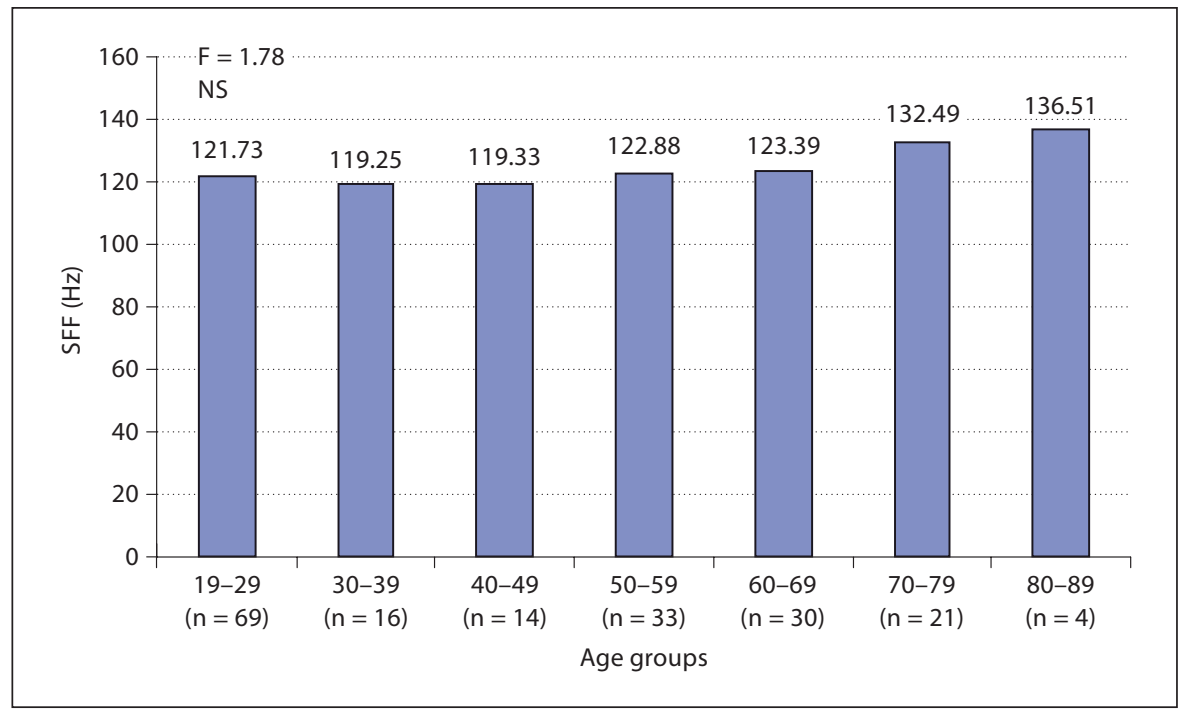

Table 2. Mean values and reference intervals of SFF (in $\mathrm{Hz}$ ) for each group

\begin{tabular}{lcccc}
\hline Groups & $\mathrm{n}$ & Mean & SD & $\begin{array}{l}\text { Reference } \\
\text { interval }\end{array}$ \\
\hline $\begin{array}{l}\text { Males, years } \\
\quad \text { Young (19-34) }\end{array}$ & 77 & 121.83 & 17.00 & $88.51-155.15$ \\
$\quad$ Middle-aged (35-59) & 55 & 120.95 & 15.78 & $90.02-151.88$ \\
$\quad$ Elderly (over 60) & 55 & 127.82 & 18.72 & $91.13-164.51$ \\
Females, years & & & & \\
$\quad$ Young (19-34) & 77 & 224.58 & 20.76 & $183.89-265.27$ \\
$\quad$ Middle-aged (35-59) & 55 & 196.31 & 16.35 & $164.26-228.36$ \\
$\quad$ Elderly (over 60) & 55 & 178.92 & 19.71 & $140.29-217.55$ \\
\hline
\end{tabular}

acoustic analysis system with robust hardware for data acquisition complemented by versatile suite of software available for speech and voice analysis. A DAT recorder was coupled to the CSL, and SFF values for each participant were analyzed individually at a $44.1 \mathrm{kHz}$ sampling rate with 16 bits of resolution.

Specifically, the voiced periodic sounds in the speech sample were extracted selectively, the fundamental frequency was numerically expressed and processed using the Frequency Analysis Program (Kay Elemetrics), which is core software for the CSL, and the mean values were obtained. At the time of analysis, each data set was carefully examined for occasional reading of spurious high or low frequency. Data analysis for all participants was conducted by one of the authors (M.N.).

\section{Reliability}

To examine the test-retest reliability of SFF measurement in this study, the 77 young female participants were asked to read the passage aloud again, according to the above-described procedure, and the speech sample was recorded. This was done at least 1 day after the original reading. The mean interval between the two recordings was 3.4. Data analysis for all participants was conducted by one of the authors (M.N.). The Pearson's product-moment correlation coefficients between original and repeated sampling of these measures was remarkably high $(\mathrm{r}=0.956, \mathrm{t}=28.39, \mathrm{p}<$ 0.001). The difference between the two mean SFFs was only 0.97 $\mathrm{Hz}$. Separately from this study, the SFFs of 77 age-matched young females were determined according to the same procedure and compared. The difference in mean SFF between these groups was only $0.1 \mathrm{~Hz}(224.58 \mathrm{~Hz}$ for the original data and 224.68 for the additional data).

\section{Results}

The mean and standard deviation of the SFF values of participants were calculated for males and females of each age group, and are presented in Hertz in table 2. In males, the difference in the measured values in the young and middle-aged groups was very small. However, the value in the elderly group was higher than those in the young and middle-aged groups by approximately $6-7 \mathrm{~Hz}$. A one-way ANOVA showed no significant differences among the three age groups (d.f. $=2, \mathrm{~F}=2.70, \mathrm{p}=0.07$ ).

All male participants were grouped according to age, by decades (19-year-old participants were included in the group in their 20s). Comparison of the mean SFFs calculated for the eight groups (for decade groups from the $20 \mathrm{~s}$ through the 80s) showed only small differences in the values of the age groups from the 20s through the 60 s, but the mean value tended to increase in the groups in their 70s and older (fig. 1). A one-way ANOVA showed no sig- 
Fig. 2. Correlation between age and SFF in male speakers.
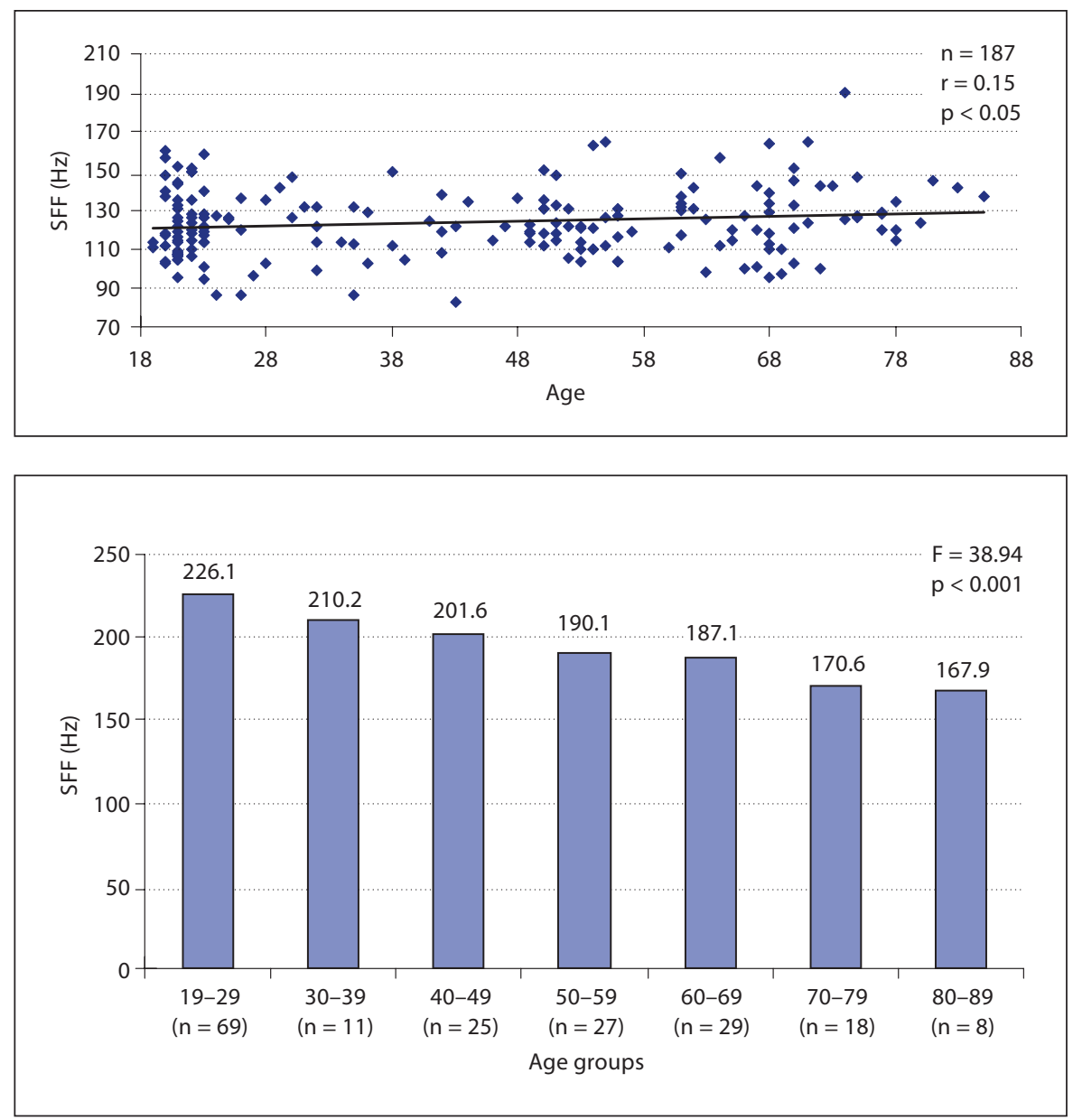

Fig. 3. Comparison of mean SFFs among age groups (female speakers).

nificant differences among the eight groups (d.f. $=6, \mathrm{~F}=$ $1.78, \mathrm{p}=0.11$ ). In addition, a post-hoc test (Scheffé's $\mathrm{F}$ ) showed no significant differences among all groups. A weak positive correlation was observed between age and SFF in all males (fig. 2) (Pearson's product-moment correlation coefficient; $\mathrm{t}=2.04, \mathrm{r}=0.15, \mathrm{p}<0.05$ ).

In females, the value was the highest in the young group, followed in order by the middle-aged group and the elderly group (table 2). A significant difference, at $\mathrm{p}<$ 0.001 , was demonstrated by a one-way ANOVA (d.f. $=2$, $\mathrm{F}=94.77$ ). When a post-hoc test (Scheffé's F) was conducted, significant differences, at $\mathrm{p}<0.001$, were observed between the young and middle-aged groups, between the young and elderly groups, and between the middle-aged and elderly groups.

All female participants were also grouped according to age by decades (19-year-old participants were included in the group in their 20s). Comparison of the mean SFFs calculated for the eight groups showed markedly lower values in the groups in their 30s and 40s than in the participants in their 20s, and a tendency for an age-related decrease was observed in all age groups through the 80s (fig. 3). A one-way ANOVA revealed significant differences at $\mathrm{p}<0.001$ (d.f. $=6, \mathrm{~F}=38.94$ ). When a post-hoc test (Scheffé's F) was conducted, significant differences were observed between the 20s group and the 40s-80s groups $(\mathrm{p}<0.001)$. Also, significant differences was observed between the 30 s group and the 70 s and 80 s groups $(\mathrm{p}<0.001)$. Furthermore, significant differences were observed between the 40 s group and the 70 s and 80 s groups $(\mathrm{p}<0.001)$. No significant difference was observed among the other age groups. A moderate negative correlation was observed between age and SFF in all females (fig. 4) (Pearson's product-moment correlation coefficient; $\mathrm{t}=$ $-15.26, \mathrm{r}=-0.75, \mathrm{p}<0.001)$.

Table 2 shows the reference intervals for males and females of each age group as the mean \pm 1.96 standard deviation. 
Fig. 4. Correlation between age and SFF in female speakers.

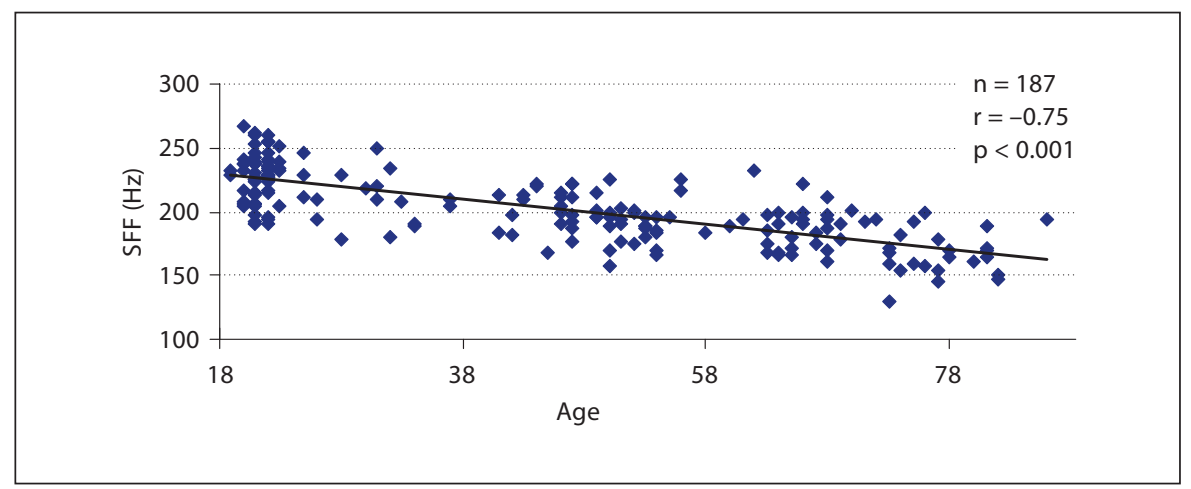

\section{Discussion}

In the present study, statistical analysis showed no significant difference in SFF values among the three age groups, and only a weak correlation between age and SFF was observed in males. When the participants were grouped according to age by decades, those in their $20 \mathrm{~s}$ through 60 s exhibited very small changes in SFF. When compared with participants in their 20s, however, the values for the age groups in their 70 s and 80 s were higher by 10.8 and $14.8 \mathrm{~Hz}$, respectively. These findings suggested that the SFF of males shows only very small changes until the seventh decade, and tends to increase slightly at age 70 years or older.

With respect to the degree of increase in SFF with aging, the classic studies by Mysak [1] and Hollien and Shipp [6] found increases of $15 \mathrm{~Hz}$ or more, while the recent studies by Pegoraro Krook [4] and Brown et al. [8] both reported an increase of less than $15 \mathrm{~Hz}$. The results of our present study support the view that the degree of increase is not more than $15 \mathrm{~Hz}$.

Hollien and Shipp [6], Pegoraro Krook [4] and Brown et al. [8] reported the SFF of males to exhibit a certain pattern characterized by transient decreases in middle age and an increase with advancing age. However, such a tendency was not observed in our study. In other words, the age-related SFF change in males was one-directional.

Consistent with a large number of previous reports [3, 4, 7-16], our results showed that SFF in females decreases in association with aging. However, the range of decrease in SFF was larger than conventionally reported. The SFF of participants in their 20s, which was used as the baseline for determining degree of change, was $226.1 \mathrm{~Hz}$. According to previous reports, the fundamental frequency of the voice in young Japanese female participants is around $225-226 \mathrm{~Hz}$ [24-26], and our results are consistent with this value. Therefore, our SFF value for participants in their 20s is considered to be valid. As mentioned in the section on reliability in the 'Methods', the validity of the value was also supported by the very similar results obtained from a separate study of 77 young females. From this baseline value, the participants in their $50 \mathrm{~s}, 60 \mathrm{~s}, 70 \mathrm{~s}$ and 80 s showed decreases of $36.0,39.0,55.5$ and $58.2 \mathrm{~Hz}$, respectively. When compared with prior reports using a cross-sectional design, the degree of SFF decrease was larger. Russell et al. [14], using a longitudinal design, reported that the value for participants in their late 60s was lower, by $47.8 \mathrm{~Hz}$, than that for participants in their $20 \mathrm{~s}$. This extent of decrease is similar to that of the present study. Thus, our results suggest that the SFF decrease in females may in fact be larger than has been suggested by conventional cross-sectional studies. However, it will be necessary to further investigate the effects of various factors, such as smoking, on SFF.

When presenting a model of male-female coalescence, Hollien [27] emphasized that SFF increases dramatically with aging in males, while the change in females is small. Our results contradict this hypothesis. In other words, SFF exhibited a relatively small change with aging in males but changed dramatically in females. This is the most surprising result of our study.

Another interesting finding obtained in our present study was the tendency in females for an obvious decrease to occur even in the 30 s, as compared with the 20 s. According to many previous studies of age groups using a cross-sectional design, SFF decreases in women after the age of 40 years [3, 4, 8-11, 13, 14].

Stoicheff [12], Pegoraro Krook [4], Morgan and Rastatter [3], and Awan [16] reported that female SFF decreases with age, but tends to increase transiently in the 70s or 80 s. However, such a tendency was not observed in the 
present study, as female SFF decreased with age in a onedirectional manner.

The fundamental frequency of the voice is largely determined by changes in the physical properties of the vocal folds, such as length, tension, and mass per unit area $[28,29]$. As the morphology of the larynx changes with age, the physical properties of the factors that determine fundamental frequency are also believed to change [29, 30]. Mueller et al. [31] reported the morphological characteristics of the aged male to include bowing, atrophy, and cordal sulcus of the vocal folds. Seventy-five percent of their older group exhibited an arrowhead configuration of the rima glottidis that was not found in their younger group. It is likely that changes in both the lamina propria and the vocalis muscle contribute to this bowing of the vocal folds and to the vibratory patterns of the vocal folds $[28,29]$. In general, changes in both the lamina propria and the vocalis muscle are greater in the male than in the female [7, 29, 32-36]. Some investigators have inferred that these changes might account for the perceptual increases in the pitch of the voice [29-31]. However, the results of the present study showed poor age-related changes in SFF for the males, and although histological changes may occur in the larynx of some aging males, histological changes do not necessarily affect the fundamental frequency of the voice.

A number of investigators have attempted to interpret the SFF change in females from the perspective of a relationship with the hormonal effects associated with menopause $[4,12,26,29,30,37,38]$. Many have hypothesized that the postmenopausal decrease in secretion of the female hormone estrogen induces morphological changes in the larynx, thereby leading to a decrease in fundamental frequency. Honjo and Isshiki [7] suggest that edema of the vocal folds in women may be due to general endocrine changes after menopause. Hirano et al. [32] hold a similar view and assume that the increased volume of the vocal folds induced by edema leads to decreased vibratory periods of the vocal folds. Although the menopause-associated female hormone decrease is marked and universal, the present results do not support the timing of the SFF decrease as conclusively occurring 'after menopause' for the following reasons: (1) a decrease in SFF is already observable in the 30 s, and (2) this decrease continues even after age 60 , which is assumed to be the postmenopausal period. The findings of the present study, which demonstrated that the SFF decrease in females starts in the $30 \mathrm{~s}$ and amounts to $24.5 \mathrm{~Hz}$ by the $40 \mathrm{~s}$, was surprising and unlike the results of any previous reports. In conclusion, the result of the present study indicate that reduction in
SFF may not be due solely to changes during menopause, but may occur in relation to premenopausal changes as suggested by Awan [16]. In addition, factors such as general lifestyle and diet may also contribute to changes in SFF with age [39].

For males, Titze [29] speculated that decreased secretion of the male hormone testosterone leads to a reduction in muscular tissues, thereby increasing SFF. While female hormone levels decrease dramatically in all women after menopause, the secretion of testosterone in males peaks in the 20s and gradually decreases thereafter. The blood concentration change in the menopausal period is gradual, however, and there is considerable individual difference in blood concentrations during the elderly period of life [40-44]. Therefore, the tendency for decreased male hormones in men is not considered to have a particularly large influence on SFF, in contrast to that of female hormones in women.

Other factors presumably associated with SFF include the effects of (1) voice training, (2) body physiology and (3) smoking. With respect to voice training, Brown et al. [8] reported that professional singers (sopranos and tenors) had significantly higher SFF levels than age-matched nonsingers. However, in young adults, Awan [45] reported no significant difference between untrained and trained groups. As to body physiology, Ramig and Ringel [2] divided 48 males into good and poor condition groups, on the basis of physical condition assessments, and then determined and compared SFF. They found that in all chronological groups (25-35, 45-55, and 65-75 years) the participants in poor condition exhibited slightly higher SFF than those in good condition. As mentioned in the 'Methods' section, voice training and body physiology were included in the selection criteria for participants in this study, but smoking was not. With regard to smoking, Gilbert and Weismer [46] and Stoicheff [12] reported that SFF in female nonsmokers was lower than that in female smokers. However, age-related changes in SFF have been reported when participants were restricted to nonsmokers $[9,12]$. Therefore, smoking may be a contributing factor for SFF, but it does not appear to be the primary factor.

Taken together, our findings indicate the reference interval for each age decade in the male and female groups to be useful not only for clinically determining SFF abnormalities and the degree of abnormality but also for detecting a variety of laryngeal diseases that can cause SFF abnormalities. For instance, vocal cord edema occurs relatively frequently in individuals with voice disorders, and a low-pitched voice is the primary symptom. Cooper 
[19] asserted that $90 \%$ of his 2,000 cases of functional and organic voice disorders could be attributed at least in part to a vocal pitch that was too low. Roy and Hendarto [47] reported that almost one half of the SFF measurements of their 40 subjects with dysphonia were considered outside the normal range. In addition, several past studies have documented the usefulness of SFF for the assessment of therapeutic effects in laryngeal diseases $[19,47,48]$. The reference intervals obtained in the present study may be used as one of the indicators for ascertaining whether or not the effects of treatments for various voice disorders reach a target level.

\section{Appendix: Questionnaire}

\section{References}

1 Mysak E: Pitch and duration characteristics of older males. J Speech Hear Res 1959;2:4654.

2 Ramig L, Ringel R: Effects of physiological aging on selected acoustic characteristics of voice. J Speech Hear Res 1983;26:27-30.

-3 Morgan EE, Rastatter M: Variability of voice fundamental frequency in elderly female speakers. Percept Mot Skills 1986;63:215218.

4 Pegoraro Krook MI: Speaking fundamental frequency characteristics of normal Swedish subject obtained by glottal frequency analysis. Folia Phoniatr Logop 1988;40:82-90.

5 Chen SH: Sex differences in frequency and intensity in reading and voice range profiles for Taiwanese adult speakers. Folia Phoniatr Logop 2007;59:1-9.
6 Hollien H, Shipp T: Speaking fundamental frequency and chronological age in males. J Speech Hear Res 1972;15:155-159.

>7 Honjo I, Isshiki N: Laryngoscopic and voice characteristics of aged persons. Arch Otolaryngol 1980;106:149-150.

$\checkmark 8$ Brown WS, Morris RJ, Hollien H, Howell E: Speaking fundamental frequency characteristics as a function of age and professional singing. J Voice 1991;5:310-315.

$\checkmark 9$ Benjamin BJ: Frequency variability in the aged voice. J Gerontol 1981;36:722-726.

10 Guimarães I, Abberton E: Fundamental frequency in speakers of Portuguese for different voice samples. J Voice 2005;19:592-606.

11 Saxman JH, Burk K: Speaking fundamental frequency characteristics of middle-aged females. Folia Phoniatr Logop 1967;19:167-172.
Gender: Male/female years)

Address:

$[$ Yes/no]

1. Are you presently healthy?
2. (Those who answered 'no' in 1): Describe your health condition specifically.

[Yes/no] $[$ Yes/no]

6. (Those who answered 'no' in 5): Why do you think your voice is different? Circle all of the applicable choices.

Cold/overworking of voice/no idea/others

Thank you very much for completing this questionnaire.

12 Stoicheff ML: Speaking fundamental frequency characteristics of nonsmoking female adults. J Speech Hear Res 1981;24:437441.

13 de Pinto O, Hollien H: Speaking fundamental frequency characteristics of Australian women: then and now. J Phonet 1982;10:367375.

14 Russell A, Penny L, Pemberton C: Speaking fundamental frequency changes over time in women: a longitudinal study. J Speech Lang Hear Res 1995;38:101-109.

-15 Awan, SN, Mueller PB: Speaking fundamental frequency characteristics of centenarian females. Clin Linguist Phonet 1992;6:249254 
16 Awan SN: The aging female voice: acoustic and respiratory data. Clin Linguist Phonet 2006;20:171-180.

-17 Shipp T, Huntington DA: Some acoustical perceptual factors in acute laryngitic hoarseness. J Speech Hear Disord 1965;30:350359.

18 Hecker MHL, Kruel EJ: Descriptions of the speech of patients with cancer of the vocal folds. Part: Measures of fundamental frequency. J Acoust Soc Am 1971;49:12751289.

-19 Cooper M: Spectrographic analyses of fundamental frequency of hoarseness before and after vocal rehabilitation. J Speech Hear Disorders 1974;39:286-297.

$>20$ Murry T, Doherty ET: Selected acoustic characteristics of pathologic and normal speakers. J Speech Hear Res 1980;23:361369.

21 Japan Tobacco Inc: National Survey of the Percentage of Tobacco Smokers. Japan Tobacco Inc., 2004.

22 Santrock JW: Adult Development and Aging. Dubuque, Brown, 1985.

23 Trubetzkoy NS: Introduction to the Principles of Phonological Descriptions. The Hague, Nijhoff, 1968

-24 Hanley TD, Snidecor JC: Some acoustic similarity among language. Phonetica 1967;17: 141-148.

25 Tsuge S, Kakami K, Fukaya M: Speaker's height, weight and voice-pitch. Onsei-gakkai-kaiho 1984;185:2-5.

$\checkmark 26$ Yamazawa H, Hollien H: Speaking fundamental frequency patterns of Japanese women. Phonetica 1992;49:128-140.
27 Hollien H: 'Old voices': what do we really know about them? J Voice 1987;1:2-17.

28 Kahane JC, Beckford NS: The aging larynx and voice; in Ripich DN (ed): Handbook of Geriatric Communication Disorders. Austin, Pro-Ed, 1991, pp 165-186.

29 Titze IR: Principles of Voice Production. Englewood Cliffs, Prentice Hall, 1994.

30 Segre R: Senescence of the voice. Eye Ear Nose Throat Monthly 1971;50:223-227.

31 Mueller PB, Sweeney RJ, Barbeau LJ: Acoustic and morphologic study of the senescent voice. Ear Nose Throat J 1985;63:71-75.

32 Hirano M, Kurita S, Nakashima T: Growth, development, and aging of human vocal folds; in Bless DM, Abbs JH (eds): Vocal Folds Physiology: Contemporary Research and Clinical Issues. San Diego, College Hill Press, 1983.

33 Sato T, Tauchi H: Age changes in human vocal fold muscles: mechanism of aging. Development 1982;18:67-74.

34 Sato K, Sakaguchi S, Hirano M: Histologic investigation of bowing of the aged vocal folds. Larynx Jpn 1996;8:11-14.

35 Kahane JC: Age related changes in the elastic fibers of the adult male vocal ligament; in Lawrence V (ed): Transcripts of the Eleventh Symposium: Care of the Professional Voice. New York, Voice Foundation, 1982.

36 Sato K: Histopathology of vocal fold atrophy. Jpn J Logop Phoniatr 2002;43:432-437.

37 Greene MC, Mathieson L: The Voice and Its Disorders. London, Whurr, 1988.

38 Rubin W: Allergic, dietary, chemical, stress, and hormonal influences in voice abnormalities. J Voice 1988;1:378-385.
39 Colton RH, Casper JK: Understanding Voice Problems: A Physiological Perspective for Diagnosis and Treatment. Baltimore, Williams \& Wilkins, 1996.

40 Kumamoto Y: Decrease of male sexual activity by aging. Jpn J Geriatr 1992;29:350-360.

41 Kato K, Motomatsu T, Akamine Y, Nawata $\mathrm{H}$, Wasada T, Oma H: Pituitary-gonadal function in the aged male. Folia Endocrinol Jpn 1977;53:1300-1309.

42 Kato K, Muta K, Ibayashi H: Re-evaluation of the parameters of human aging process: pituitary-gonadal function in elderly men. Jpn J Geriatr 1984;21:219-225.

43 Ibayashi H: Aging changes in hormone production. Jpn J Geriatr 1974;11:138-146.

44 Ibayashi H: Aging changes in hormone production. Folia Endocrinol Jpn 1983;59:17831789.

45 Awan SN: Superimposition of speaking voice characteristics and phonetograms in untrained and trained vocal groups. J Voice 1993; 7:30-37.

46 Gilbert HR, Weismer GG: The effect of smoking on the speaking fundamental frequency of adult women. J Psycholinguist Res 1974;3:225-231.

47 Roy N, Hendarto H: Revisiting the pitch controversy: changes in speaking fundamental frequency (SFF) after management of functional dysphonia. J Voice 2005; 19:582-591.

48 Roy N, Tasko SM: Speaking fundamental frequency (SFF) changes following successful management of functional dysphonia. J Speech Lang Pathol Audiol 1944;18:115120. 